Article

\title{
Adaptive Bat Algorithm Optimization Strategy for Observation Matrix
}

\author{
Zhihua Cui *, Chunmei Zhang, Yaru Zhao ${ }^{\mathbb{D}}$ and Zhentao Shi \\ Complex System and Computational Intelligent Laboratory, Taiyuan University of Science and Technology, \\ Taiyuan 030024, China \\ * Correspondence: cuizhihua@tyust.edu.cn; Tel.: +0351-699-8016
}

Received: 3 June 2019; Accepted: 23 July 2019; Published: 26 July 2019

Featured Application: This paper proposes an adaptive bat algorithm to optimize the observation matrix and achieve signal reconstruction. By introducing the theory of compressed sensing, the adaptive bat algorithm is designed to realize the signal reconstruction process. The proposed algorithm has been extensively evaluated. In particular, the effects of different algorithms on different algorithms with different signals and the effects of different transform bases on the proposed method are studied.

\begin{abstract}
Bat algorithm, as an optimization strategy of the observation matrix, has been widely used. Observation matrix has a direct impact on the reconstructed signal accuracy as a projection transformation matrix, and it has been widely used in various algorithms. However, for the traditional experimental process, randomly generated observation matrices often result in a larger reconstruction error and unstable reconstruction results. Therefore, it is a challenge to retain more feature information of the original signal and reduce reconstruction error. To obtain a more accurate reconstruction signal and less memory space, it is important to select an effective compression and reconstruction strategy. To solve this problem, an adaptive bat algorithm is proposed to optimize the observation matrix in this paper. For the adaptive bat algorithm, we design a dynamic adjustment strategy of the optimal radius to improve its global convergence ability. The results of our simulation experiments verify that, compared with other algorithms, it can effectively reduce the reconstruction error and has stronger robustness.
\end{abstract}

Keywords: adaptive bat algorithm; observation matrix; reconstruction error; signal reconstruction

\section{Introduction}

With signals, images and video data are gradually increasing in contemporary life. To reduce the cost of data storage, transmission and processing, signals are often represented with less storage space at an acceptable level of distortion. Compressed sensing, as a technique for finding sparse solutions of underdetermined linear systems, is widely used in many fields, such as image processing, medical imaging, computer science, signal processing. Particularly, compressed sensing is used in signal processing to obtain and reconstruct sparse or compressible signals. For example, studies [1-4] have shown that it is feasible to directly obtain a compressed representation with a variety of assumptions.

The sparse observation matrix plays an important role in the reconstruction algorithm. In [5], Wang et al. expounded the advantages of the compressed sensing theory in solving the problem of information redundancy for the traditional signal acquisition process. They analyzed the construction of the compressed sensing measurement matrix from four aspects. Donoho et al. [6] proposed a method for linear measurement of a given vector and returned it to the Euclidean accuracy range. Moreover, they suggested that the observation matrix should satisfy the restricted isometry property (RIP), and 
the random Gaussian matrix was employed as the usual observation matrix. Then, the discriminant rules of the observation matrix and the sparse representation transformation basis were proposed by Candès et al. [7]. Fang et al. [8] took advantage of the tail boundedness of the sub-Gaussian distribution to design the sub-Gaussian distribution sparse observation matrix. Candès et al. [9] revealed the relationship between the random Bernoulli matrix and the restricted isometry property (RIP) criterion. And to reduce storage space, Yin [10] designed a Toeplitz matrix in the RIP criterion, which was generated by a single row vector loop.

The random Gaussian observation matrix is an important method in the reconstruction process, which has received much attention and shown some advantages. Peng et al. [11] decomposed the observation matrix by using singular value and increased the observation matrix independence of each column by adopting the mean value improvement strategy. Duarte et al. [12] proposed the evaluation index between the observation matrix and transform basis, and used the eigenvalue decomposition method to optimize the random Gaussian observation matrix. Abolghasemi et al. [13] proposed the Gram matrix to make the observation matrix close to the unit matrix by optimizing iteration. Lan et al. [14] used a gradient descent method to convert the Gram matrix to the unit matrix. And in order to reduce the correlation between the observation matrix and the transform basis, the orthogonal triangulation $(\mathrm{QR})$ decomposition was proposed. According to the characteristics of the MIMO radar signal, Yu et al. [15] optimized the signal-to-interference ratio and reduced the correlation and noise.

In recent years, a large number of bio-inspired optimization algorithms have been proposed, such as the genetic algorithm (GA) [16,17], bat algorithm (BA) [18-21], differential evolution (DE) [20,21], firefly algorithm (FA) [22], artificial bee colony (ABC) [23,24], cuckoo algorithm (CS) [25-27], and so on [28-32], which have been applied to various fields, including the optimization problems [33-37], practical application problems [38-41], LEACH (Low Energy Adaptive Clustering Hierarchy) protocol optimization [42], and so on [43-46]. Particularly, Cui et al. [47] designed an optimization method based on the bat algorithm to optimize the random Gaussian observation matrix and reduced the reconstruction error.

The remainder of this paper as follows. Section 2 gives an overview of the related work and our motivations. Section 3 introduces the proposed adaptive bat algorithm (ABA) in detail and indicates the specific method flow. In Section 4, the simulation experiments are extensively tested, and the performance of this algorithm is compared. Conclusions are given in Section 5.

\section{Related Works}

To analyze the influence of the observation matrix on the algorithm, five different observation matrices and two algorithm optimized observation matrices are used for reconstructing the signal. It includes the random Gaussian observation matrix [6], random Bernoulli observation matrix [9], singular value decomposition, mean optimization random Gaussian observation matrix [11], the observation matrix optimized by the standard bat algorithm, and the observation matrix optimized by the adaptive bat algorithm.

The signal recovery, as an important process of compressed sensing, has an essential impact on reconstruction accuracy. Therefore, researchers have proposed many improvement strategies to improve the accuracy of signal recovery, which include the following four categories: (1) Greedy algorithm based on local search strategy [48,49]; (2) convex optimization algorithm based on linear programming problem [50,51]; (3) non-convex optimization algorithm based on linear programming problem [52,53]; and (4) reconstruction algorithm based on natural heuristic algorithm [54,55]. In the process of reconstruction [56], the signals can be compressed and sampled in real-time; moreover, the original signals also can be recovered by some specific reconstruction algorithms.

Orthogonal matching pursuit algorithm (OMP) [57], due to its fast reconstruction velocity and simple implementation, is widely used in the field of compressed sensing. In this paper, the OMP algorithm is employed to optimize the observation matrix. Its process is as follows: 
1. Calculate the correlation coefficient between the sensing matrix $\left(A_{C S}\right)$ and residual projection, and select the maximum correlation coefficient atomic as updated support sets.

2. Use the Schmidt orthogonalization processing for the sensing matrix to eliminate interference on subsequent atoms.

3. Least-square method is used to update residuals and obtain the reconstructed signals.

The bat algorithm can obtain better results when solving continuous optimization problems. However, for a large number of discrete problems in practical engineering, it often tends to cause slow convergence and fall into local optimum. Therefore, discrete bat algorithms have been proposed. Mirjalili et al. [58] proposed a binary bat algorithm to deal with discrete problems and introduced a binary coding strategy to update the velocity and position of bats individuals. Osaba et al. [59] proposed a discrete bat algorithm and introduced Hamming code distance to enhance the ability to learn from optimal bats. Xu et al. [60] applied the discrete bat algorithm to the flexible shop scheduling problem. To accelerate the convergence velocity of the algorithm, they also proposed to introduce the bat's position mutation strategy into the position update strategy and calculated the fitness value by using the clock algorithm.

To jump out of the local extremum solution, Liu et al. [61] introduced the Lévy flight strategy and updated the speed and position of the individual by randomly increasing the step size. Different from the above work, Xie et al. [62] directly used the Lévy flight strategy to update the speed and position. In order to enhance the local search ability, Wang et al. [63] proposed a novel variant of the bat algorithm based on global search and introduced a weighting factor. According to the requirements of numerical optimization problems, Tsai et al. [64] redefined the updated equation of velocity and position, and proposed an evolutionary bat algorithm, which makes the algorithm achieve higher accuracy in the optimization process.

In addition, for multi-objective optimization problems, many multi-objective bat algorithms have been proposed. Yang [65] proposed a multi-objective bat optimization algorithm (MOBA) to solve continuous optimization problems and introduced the weighted method into the algorithm. Wang et al. [66] proposed a multi-objective bat algorithm based on decomposition (MOBA/D), which is to decompose multiple problems into sub-problems and then reaggregate. Besides, differential evolution operators are introduced to increase the population diversity and the convergence velocity. According to the trade-off between two objectives in data mining of association rules, Heraguemi et al. [67] proposed the multi-objective bat algorithm based on NSGA-II (Non dominated sorting genetic algorithm-II), and the flight frequency and velocity of bat individuals in the algorithm are improved.

As we have known, the observation matrix is an important factor in the signal reconstruction process; however, most scholars ignore the optimization of the observation matrix. Moreover, to achieve better signal reconstruction, according to the RIP theorem, we can obtain the correlation between the transformed base and the observation matrix. However, the experiment of the influence of the choice of different transform bases on the optimization algorithm has not been found in previous works. Therefore, in order to obtain a higher reconstruction accuracy and smaller reconstruction error, an adaptive bat algorithm to optimize the observation matrix is designed. In addition, we also tested the effects of different observation matrix reconstructions and different transform based on the proposed algorithm. Finally, signal reconstruction is achieved.

\section{Adaptive Bat Algorithm Optimizes Observation Matrix}

In the field of signal processing, compressed sensing technology has been widely used as the latest technology. Since the observation matrix and reconstruction algorithm have a certain influence on the effect of signal reconstruction, this paper designs a novel observation matrix and reconstruction algorithm for this phenomenon. In order to avoid the individual falling into local extremum and achieve better reconstruction effect of the optimized observation matrix, the paper designs the comparison of different observation matrices with different algorithms, so as to compare the signal reconstruction effect. 
To enhance the reconstruction accuracy and minimize the reconstruction error, an adaptive bat algorithm is proposed to optimize the observation matrix. In this section, compressive sensing theory and the search method of ABA are introduced in detail; additionally, the strategy of optimizing the observation matrix based on the adaptive bat algorithm is described.

\subsection{Compress Sensing}

In this subsection, the process of compressed sensing is introduced. Firstly, the sparse signals are calculated as follows:

$$
X=\Psi \Theta
$$

where $\Theta\left(\Theta \in R^{N \times 1}\right)$ represents a sparse representation of the signal $X$ on the transform base $\Psi$, and the length of signal $X$ is $N$.

Then, observation signal (where, the dimension of observation signal is $M$ ) can be compressed with Equation (2).

$$
Y=\Phi \Psi \Theta=A_{C S} \Theta
$$

where $Y$ represents the observation signals, $\Phi \in R^{M \times N}$ is observation matrix, $A_{C S}=\Phi \Psi$ is sensing matrix.

Finally, the reconstruction process model is described as shown in Equation (3).

$$
\left\{\begin{array}{l}
\min _{\Theta}\|\Theta\|_{0} \\
\text { s.t. } Y=A_{C S} \Theta
\end{array}\right.
$$

where $\|\Theta\|_{0}$ represents the $l_{0}-$ norm.

\subsection{The Search Methods of Adaptive Bat Algorithm}

To improve the global search ability, an adaptive bat algorithm is designed in this paper. The main search method for ABA is that firstly, the optimization center and a preferred radius for the global optimal position are determined; then, update the velocity and position for individuals within the search radius, inherit the information of the previous generation and the optimal position, and accelerate the search of the global optimal solution by the algorithm. For an individual outside the optimization radius, since the individual is not closest to the optimal solution, the individual is randomly searched within the defined domain. This strategy avoids the individual falling into local extremum and increases the global search ability of the algorithm. However, when the position of the optimal global solution is unknown, the optimal position of each generation of bat individuals is taken as the optimization center, and the optimization radius of the optimization center is calculated. Finally, when the individual does not have a search center, a random search is used to increase the global search ability of the population. Next, the search methods of ABA are introduced.

For the individuals within the optimization center, velocity and position updates are as follows:

$$
\begin{gathered}
v_{i}^{t}=v_{i}^{t-1}+\left(x_{i}^{t-1}-x_{g b e s t}\right) \times f_{i} \\
x_{i}^{t}=x_{i}^{t-1}+v_{i}^{t}
\end{gathered}
$$

where $x_{i}^{t}$ represents the position of bat $i$ at time $t, v_{i}^{t}$ represents the velocity of bat $i$ at time $t, x_{\text {gbest }}$ represents the global optimal solution, $f_{i}$ represents the pulse frequency.

The optimization process of the observation matrix is based on the transformation base $\Psi$ and OMP reconstruction algorithm. Equation (6) is considered the objective function (fitness).

$$
f(x)=\left\|A_{C S} \alpha-Y\right\|_{2}^{2}
$$


where $f(x)$ represents the fitness value function, $Y$ represents the observed signal, $\alpha$ represents the sparse representation of the original signal on the transformation base. And the individuals with the minimum fitness value are selected as the optimal solution.

Additionally, the dynamic adjustment of the radius is achieved by the following:

$$
\text { radius }=\frac{f_{x_{\text {gbest }}^{t}}}{\sum_{i=1}^{P} f_{x_{i}^{t}}}
$$

where $x_{\text {gbest }}^{t}$ represents the optimal position at time $t, P$ represents individual number, radius represents the search radius. Apparently, the radius decreases as the objective function decreases.

To reduce the reconstruction failures probability, the flow of the proposed adaptive bat algorithm search is shown in Figure 1.

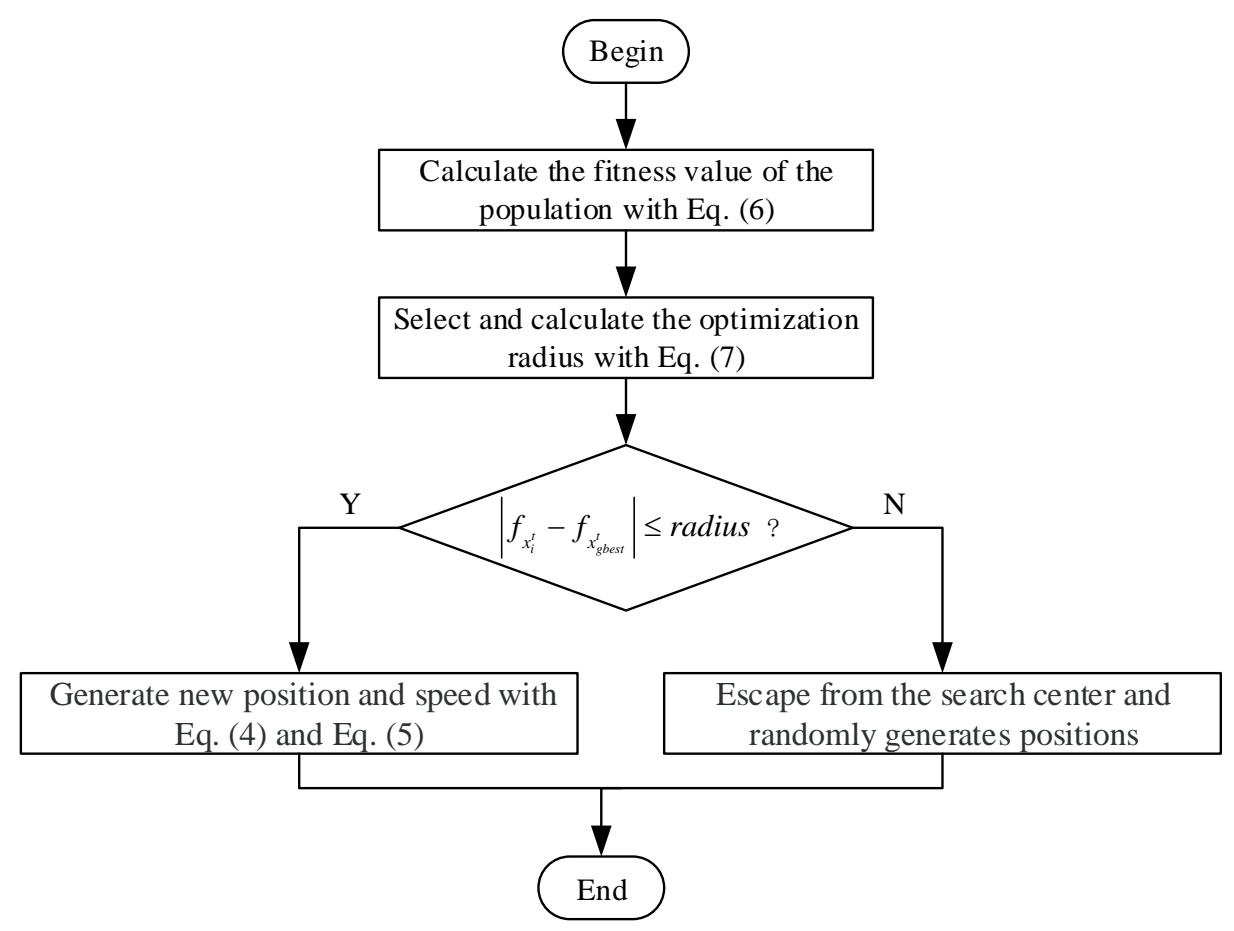

Figure 1. The flow chart of the adaptive bat algorithm search.

In this process, a new optimization center and radius are added in each generation of bat algorithm, and the local and global search ability is enhanced.

\subsection{Observation Matrix Optimization Based on Adaptive Bat Algorithm}

\subsubsection{Population Initialization and Coding Strategy}

In this section, the bat algorithm is used to optimize the observation matrix to determine the position and velocity of bats individual, and Gaussian random matrices (the size $M \times N$ ). In addition, according to the fitness values, the better individuals are selected (the fitness values stored in the last dimension of the matrix, e.g., Equation (8)). At the same time, the new position is disturbed in the 
first $M \times N$ dimension and the $M \times N+1$ dimension is updated. In this way, the most appropriate observation matrix and corresponding error for different signals is selected.

$$
x=\llbracket\left[\begin{array}{c}
a_{1} \\
a_{2} \\
a_{3} \\
a_{4} \\
\cdots \\
a_{M N}
\end{array}\right]\left[\begin{array}{c}
b_{1} \\
b_{2} \\
b_{3} \\
b_{4} \\
f_{x_{1}^{t}}
\end{array}\right]\left[\begin{array}{c}
c_{1} \\
c_{2} \\
c_{3} \\
b_{M N} \\
f_{x_{2}^{t}}
\end{array}\right]\left[\begin{array}{c}
c_{4} \\
\ldots \\
c_{M N} \\
f_{x_{3}^{t}}
\end{array}\right] \ldots
$$

where $x$ represents the bat individual, $M \times N+1$ represents the individual dimension.

\subsubsection{Define Population Interval}

The foraging behavior of bats is to find relatively better food in an area; the area setting can ensure that bats do not fly out of this area during the foraging process. In this algorithm, the initial position of the bat population is the Gaussian random observation matrix, which obeys the standard normal distribution, and the algorithm defines the domain interval as $[-3,3]$.

\subsection{The Design of Adaptive Bat Algorithm Optimal Observation Matrix}

In this section, the flow chart of the adaptive bat algorithm based on observation matrix optimization is described. The adaptive bat algorithm, to realize the observation matrix optimization search method by dynamically adjusting the size of the optimization radius, is as shown in Algorithm 1.

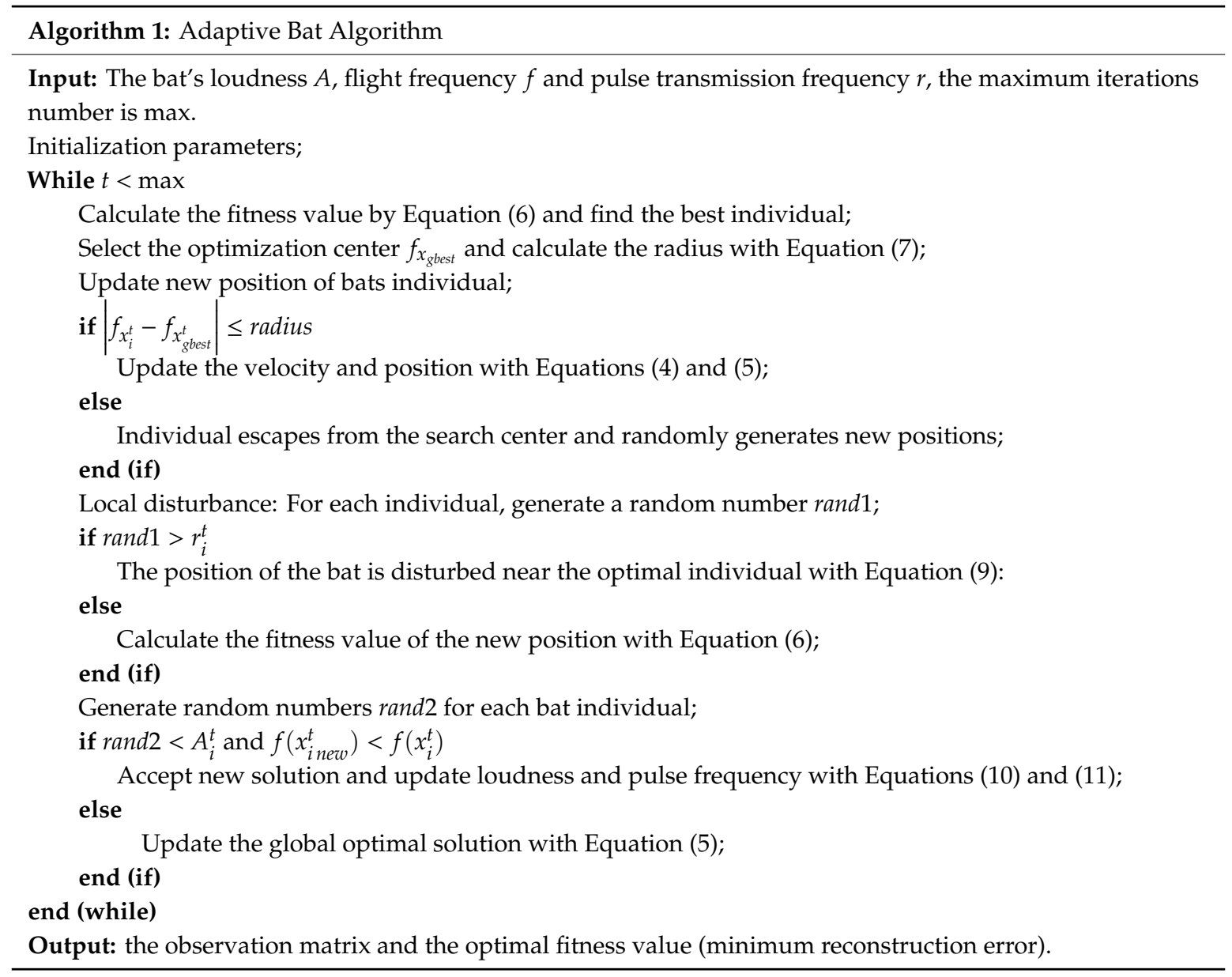


Among them, the position of the bat individual is disturbed near the optimal individual with Equation (9):

$$
x_{\text {inew }}^{t}=x_{\text {gbest }}+\rho \overline{A^{t}}
$$

where $\rho \in[-1,1], \overline{A^{t}}$ represents the average pulse loudness of bat individual at time $t$.

And then, the updated pulse frequency and loudness equation are as follows:

$$
\begin{gathered}
r_{i}^{t+1}=r_{i}^{t}\left(1-e^{-\gamma t}\right) \\
A_{i}^{t+1}=\psi A_{i}^{t}
\end{gathered}
$$

where $\gamma$ represents the growth rate of pulse transmission frequency, and the value is an integer with greater than $0, \psi$ represents the decrease rate of the pulse frequency.

Algorithm 2 describes the process of the proposed adaptive bat algorithm for signal reconstruction based on transform base and OMP algorithm. Among them, the main process of reconstructing is introduced in Algorithm 2.

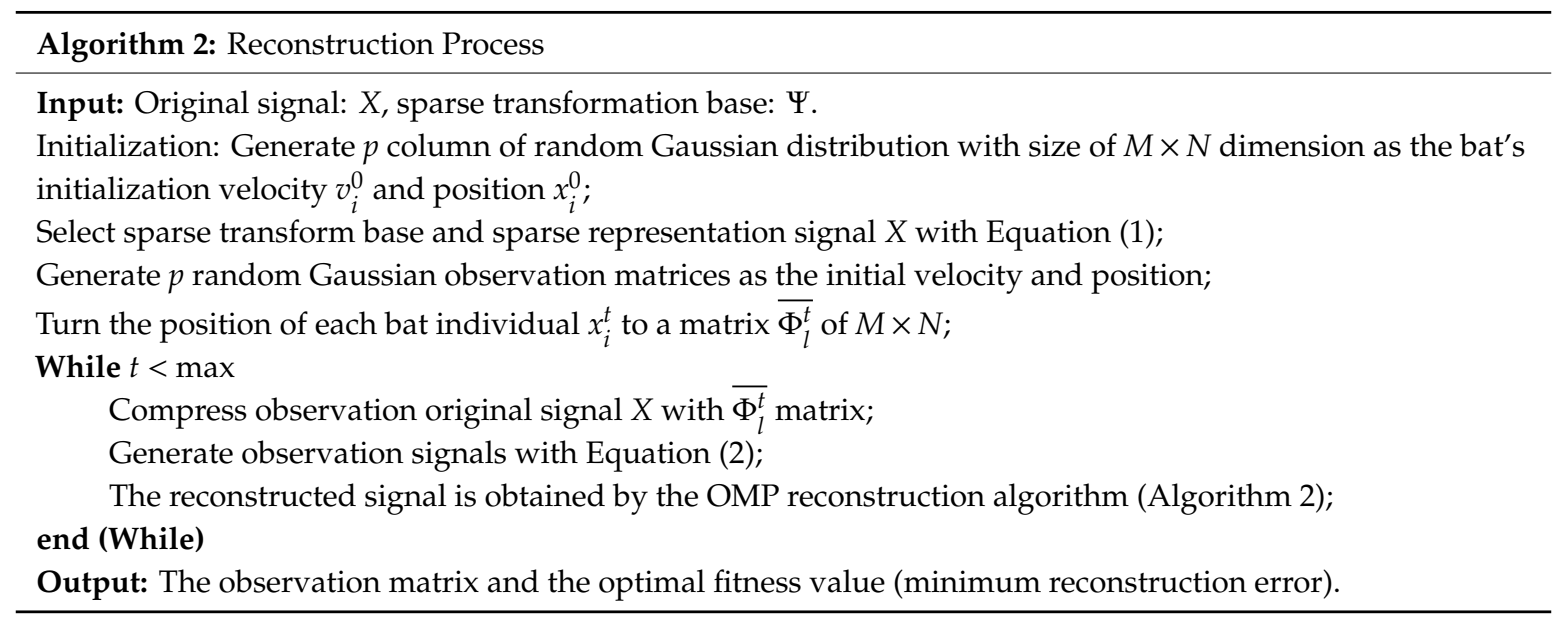

\subsection{The Flow Chart of Adaptive Bat Algorithm Optimal Observation Matrix}

In the previous section, we proposed an adaptive bat algorithm to optimize the observation matrix. Firstly, the algorithm parameters and reconstructed signal are initialized. Secondly, the compression projection is carried out based on the relevant principles of compressed sensing, and signal reconstruction is realized. Thirdly, according to the search method of our adaptive bat algorithm, the optimization radius and new position are constantly updated. Finally, the optimal global solution is obtained. In our model, the observation matrix is optimized and the reconstruction error value is updated by generating the optimal position and the optimal fitness value. The process of the adaptive bat algorithm optimal observation matrix is shown in Figure 2. 


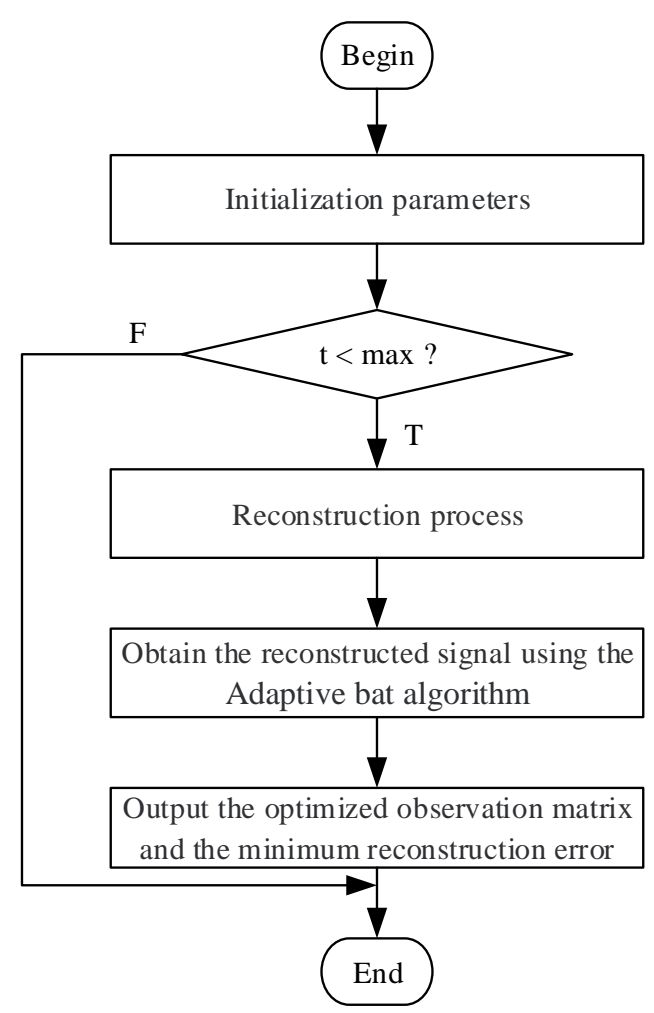

Figure 2. The flow chart of adaptive bat algorithm optimal observation matrix.

\section{Experimental Simulation}

To verify the performance optimization of the observation matrix by the ABA, this paper conducted two groups of experiments: Signal reconstruction experiment and the selection of transform bases for the ABA to optimize the performance of the observation matrix. All simulation experiments were carried out in MATLAB environment.

\subsection{Experimental Design}

\subsubsection{Comparison Algorithm}

In this paper, the effect of signal reconstruction is compared with the following observation matrices:

- Gaussian random observation matrix (Gaussian),

- Bernoulli random observation matrix (Bernoulli),

- Singular value decomposition and the average value of optimization random gaussian observation matrix [37] (SVD-M),

- Standard bat algorithm (BA) optimization random Gaussian observation matrix [36],

- Adaptive bat algorithm optimization the observation matrix (ABA).

\subsubsection{Signal Test}

In the signal reconstruction experiment, four unidimensional sparse signals are selected, respectively, as shown in Table 1. 
Table 1. Sparse signals.

\begin{tabular}{l}
\hline The Test of Sparse Signal: \\
\hline$S_{1}=0.3 \cos \left(2 \pi f_{1} T_{s} t_{s}\right)+0.6 \cos \left(2 \pi f_{2} T_{s} t_{s}\right)+0.1 \cos \left(2 \pi f_{3} T_{s} t_{s}\right)+0.9 \cos \left(2 \pi f_{4} T_{s} t_{s}\right)$ \\
\hline$S_{2}=6 \cos \left(80 \pi f_{1} T_{s} t_{s}\right)-7 \cos \left(10 \sin \left(\pi f_{2}\right) T_{s} t_{s}\right)-3 \cos \left(20 \sin \left(\pi f_{4} T_{s} t_{s}\right)\right)$ \\
\hline$S_{3}=\sin \left(11 \pi f_{1} T_{s} t_{s}\right)+\cos \left(17 \pi f_{2} T_{s} t_{s}\right)-0.4 \cos \left(6 \pi f_{3} T_{s} t_{s}\right)$ \\
\hline$S_{4}=32 \sin \left(4 \pi f_{1} T_{s} t_{s}\right)+60 \cos \left(\pi f_{2} T_{s} t_{s}\right)-2.2 \sin \left(60 \pi f_{3} T_{s} t_{s}\right)+0.2 \sin \left(2 \sin \left(16 \pi f_{4}\right) T_{s} t_{s}\right)$ \\
\hline \\
represents sampling frequency, $t_{s}=\frac{1}{F_{s}}$ represents sampling interval, $T_{s}=1: N$ represents sampling sequence, \\
$f_{1}, f_{2}, f_{3}$, and $f_{4}$ represent signal frequency, respectively, rate $=\frac{M}{N}$ represents the compression ratio, $K$ represents \\
he sparse degree and $K=\left\lceil\frac{M}{\log N}\right]$.
\end{tabular}

\subsubsection{Parameter Setting}

In the experiment, some parameters are shown in Table 2.

Table 2. Parameter setting.

\begin{tabular}{cccc}
\hline Parameter & Value & Parameter & Value \\
\hline signal length $N$ & 256 & signal frequency $f_{1}$ & $50 \mathrm{~Hz}$ \\
compression rate & $0.3,0.35,0.4,0.45,0.5$ & signal frequency $f_{2}$ & $100 \mathrm{~Hz}$ \\
sampling frequency $F_{S}$ & 800 & signal frequency $f_{3}$ & $200 \mathrm{~Hz}$ \\
population size $P$ & 100 & signal frequency $f_{4}$ & $400 \mathrm{~Hz}$ \\
\hline
\end{tabular}

\subsection{Signal Reconstruction Experiment}

In the signal reconstruction experiment, four sparse unidimensional signals $\left(S_{1}, S_{2}, S_{3}\right.$ and $\left.S_{4}\right)$ are selected. And the ABA performance is tested by comparing the error values of five observation matrices and the curve fluctuation amplitude with different compression ratios.

\subsubsection{Evaluation Index Design}

Reconstruction error $(R E)$ is used to evaluate the quality of reconstructed signals, and Equation (12) is used to calculate. The smaller the $R E$ value is, the smaller the reconstruction error is and the better the reconstruction accuracy is.

$$
R E=\frac{\left\|X-X^{*}\right\|_{2}}{\|X\|_{2}}
$$

where $X$ represents the original signal, $X^{*}$ represents the reconstructed signal.

\subsubsection{Signal Reconstruction Experiment and Analysis}

Figure 3 shows the performance comparison of four signals reconstructed by the adaptive bat algorithm with a compression ratio of 0.3 . And the randomly generated 30 observation matrices corresponding to the various observation matrices are used for the test experiments. The trend of the original signal and the recovered signal are compared by calculating the mean and variance. As can be seen from Figure 3, the ABA has a better reconstruction effect on the original signal. In addition, the proposed algorithm can effectively reconstruct the original signal in a relatively uniform area and achieve stable characteristics. Therefore, the ABA optimized observation matrix below is introduced. However, since the test process of the signal reconstruction has not selected a good observation matrix, there will be a case where the algorithm fluctuates in the signal reconstruction effect. This also reflects the innovation of the paper using the proposed algorithm to optimize the observation matrix. The difference in reconstruction accuracy for each algorithm and different observation matrices is shown in Figures $4-7$. 


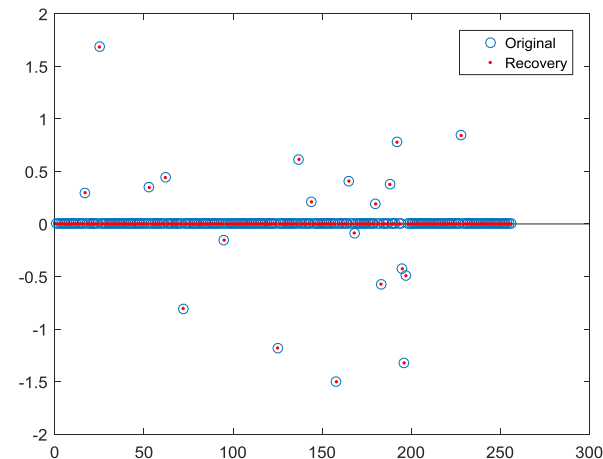

(a)

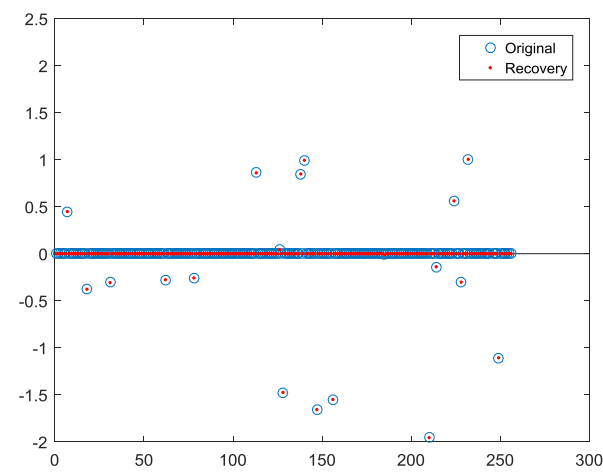

(c)

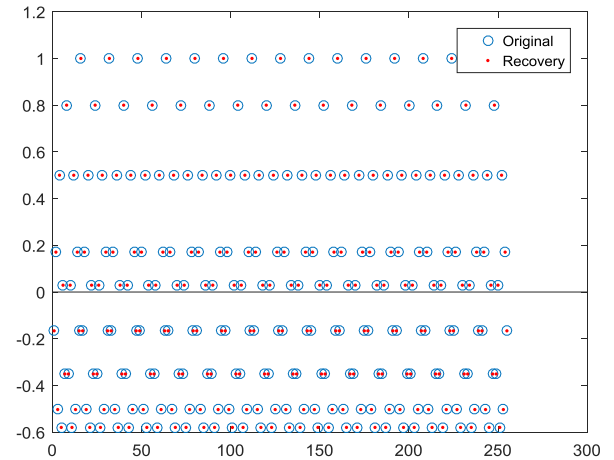

(b)

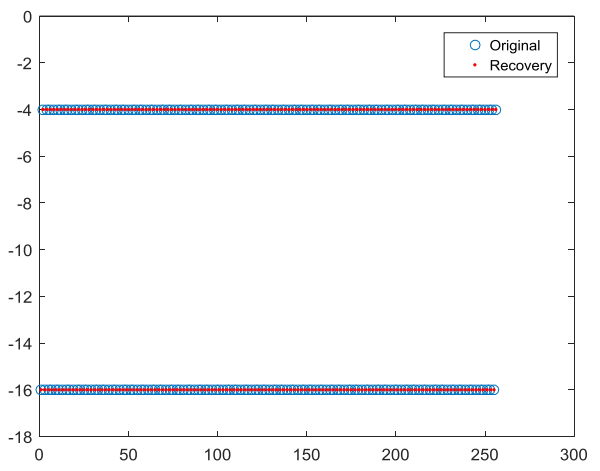

(d)

Figure 3. Reconstruction effect diagram for different signals: (a) Signal 1; (b) Signal 2; (c) Signal 3; (d) Signal 4.

We can see from Figures 4-7 that it is feasible for the ABA to optimize the observation matrix in terms of improving the stability of the observation matrix or enhancing the reconstruction accuracy of the signal. Therefore, the observation matrix optimized by ABA is more reliable.

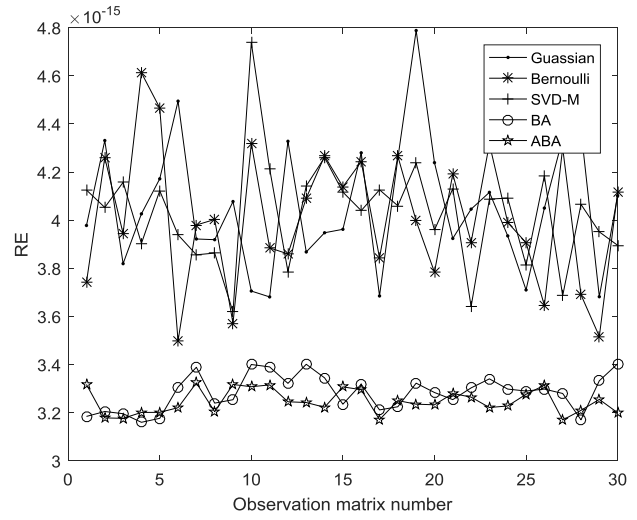

(a)

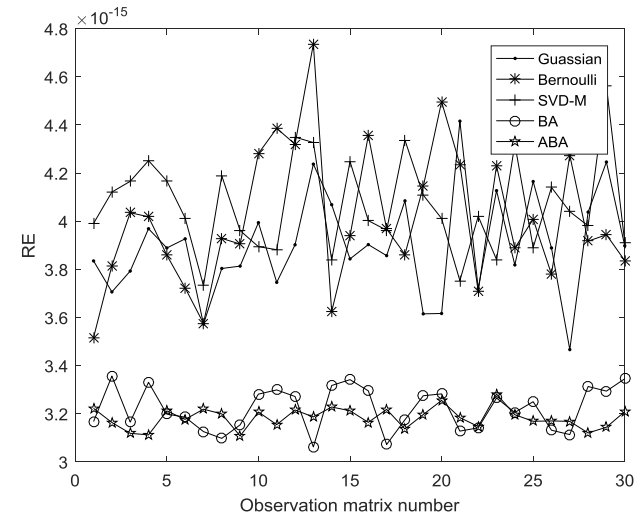

(b)

Figure 4. Cont. 


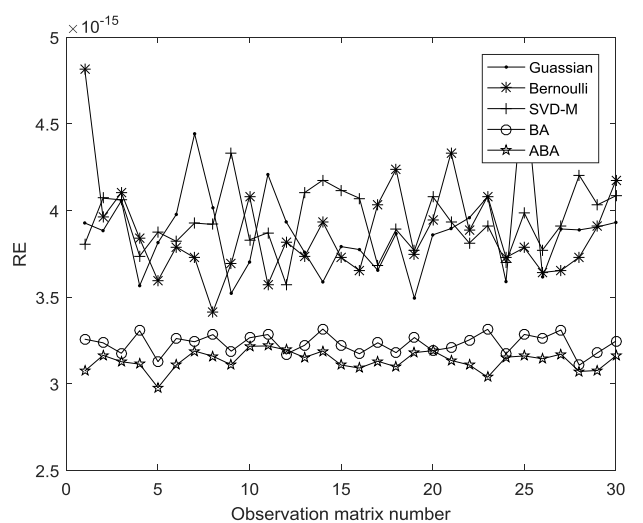

(c)

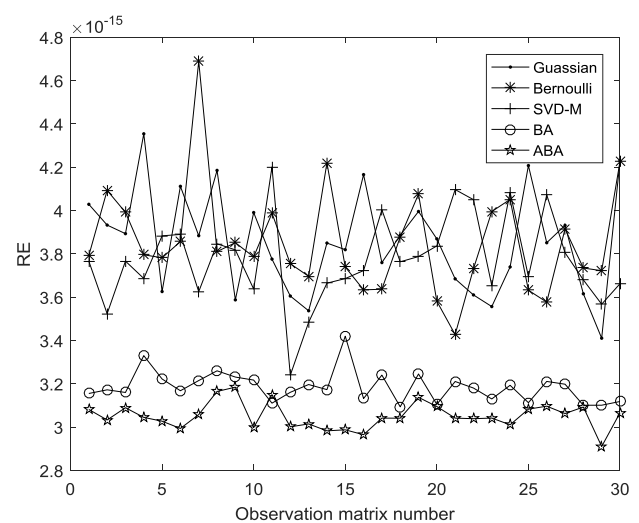

(d)

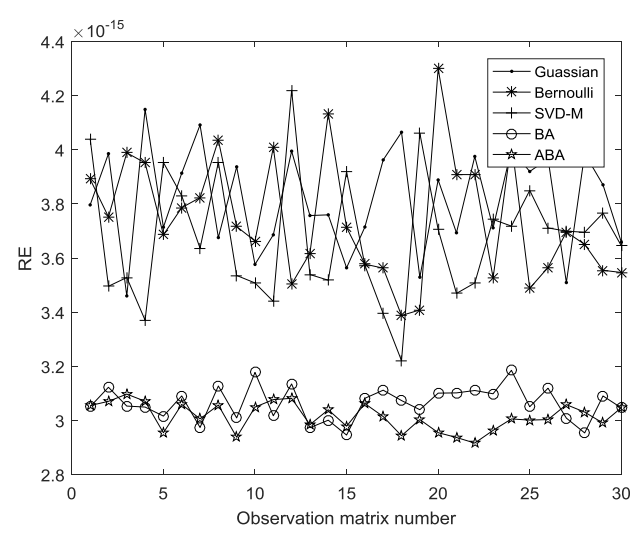

(e)

Figure 4. Reconstruction error of each algorithm to signal-1 with different compression ratio: (a) Rate $=0.3 ;(\mathbf{b})$ Rate $=0.35 ;(\mathbf{c})$ Rate $=0.4 ;(\mathbf{d})$ Rate $=0.45 ;(\mathbf{e})$ Rate $=0.5$.

Figure 4 shows the reconstructed error values obtained for the $S_{1}$ with different compression ratios. It can be seen from Figure 4 that the reconstructed error curves of Gaussian, Bernoulli, and SVD-M have large amplitude fluctuations and poor error values. However, the BA and ABA have smaller error values and a minor fluctuation. This also proves that the reconstruction effects of $B A$ and ABA are better than observation matrixes.

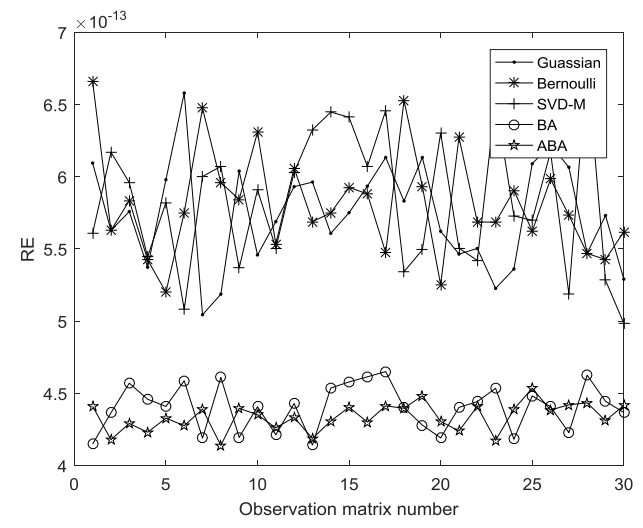

(a)

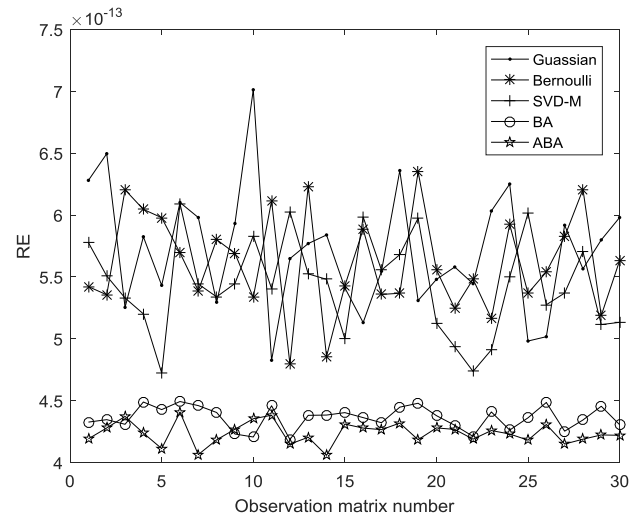

(b)

Figure 5. Cont. 


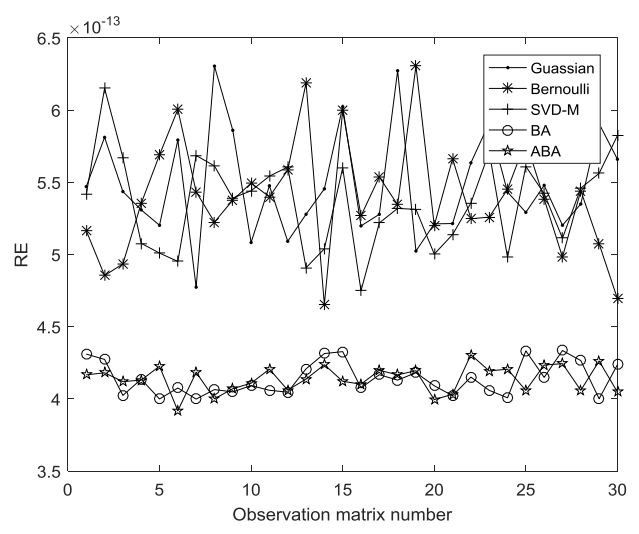

(c)

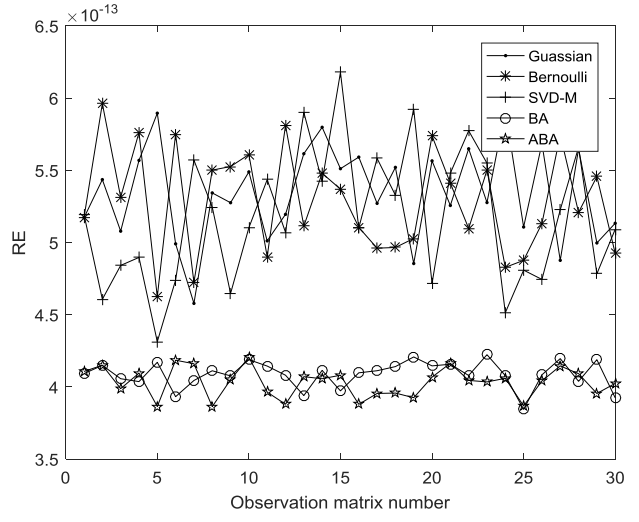

(d)

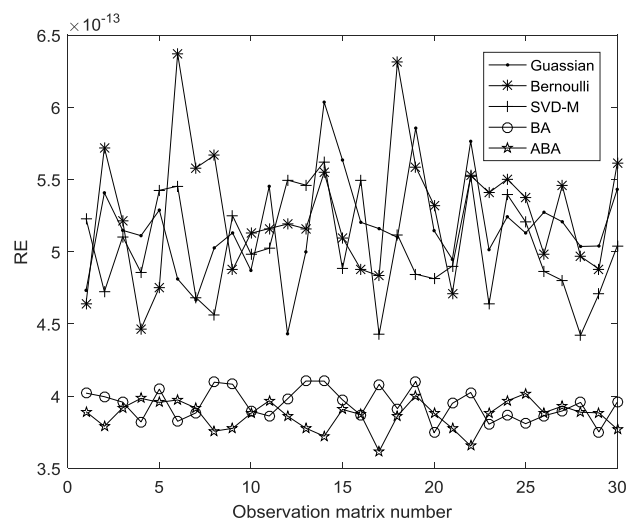

(e)

Figure 5. Reconstruction error of each algorithm to signal-2 with different compression ratio: (a) Rate $=0.3 ;(\mathbf{b})$ Rate $=0.35 ;(\mathbf{c})$ Rate $=0.4 ;(\mathbf{d})$ Rate $=0.45 ;(\mathbf{e})$ Rate $=0.5$.

For the $S_{2}$ signals, Figure 5 depicts a reconstruction error plot of the observation matrix and algorithms with different compression ratios. It can be seen from Figure 5 that the reconstruction error of Gaussian, Bernoulli, and SVD-M fluctuates greatly and the stability of reconstruction error is poor. Conversely, BA and ABA have better reconstruction error values and the variation is stable.

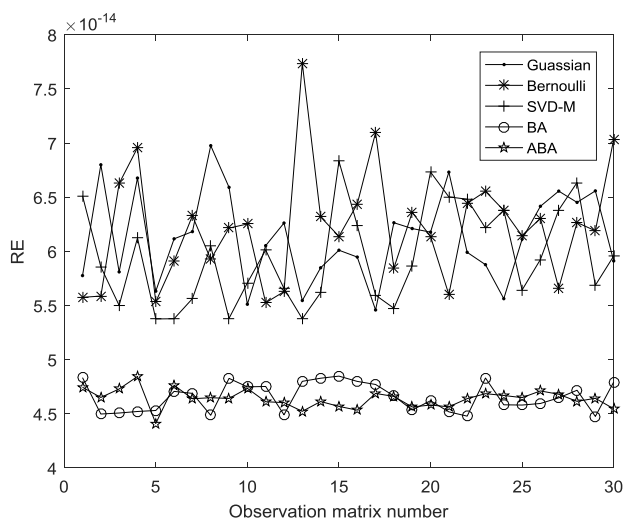

(a)

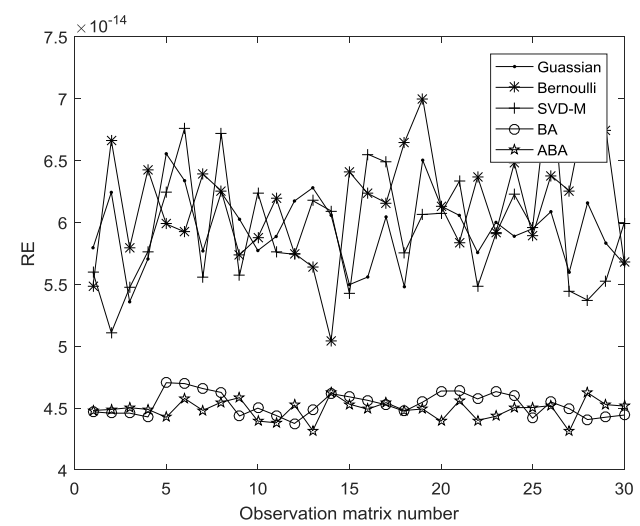

(b)

Figure 6. Cont. 


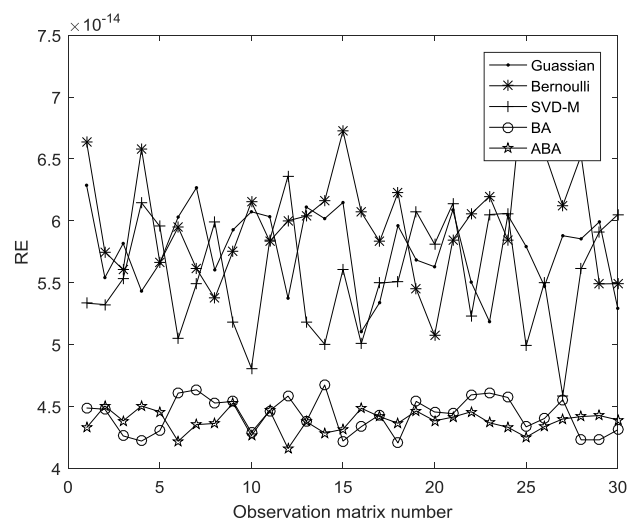

(c)

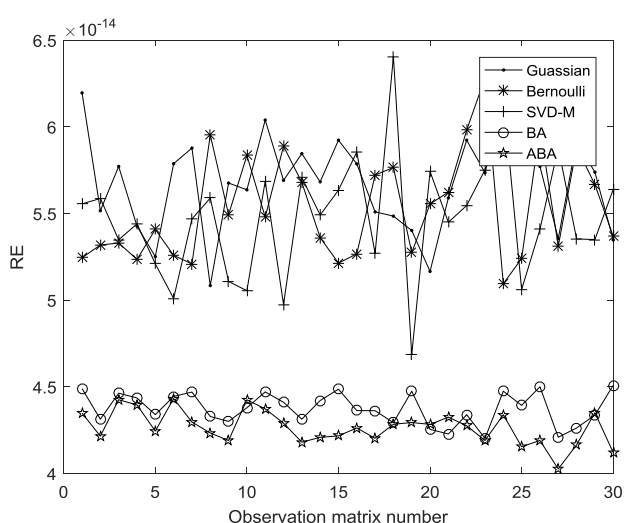

(d)

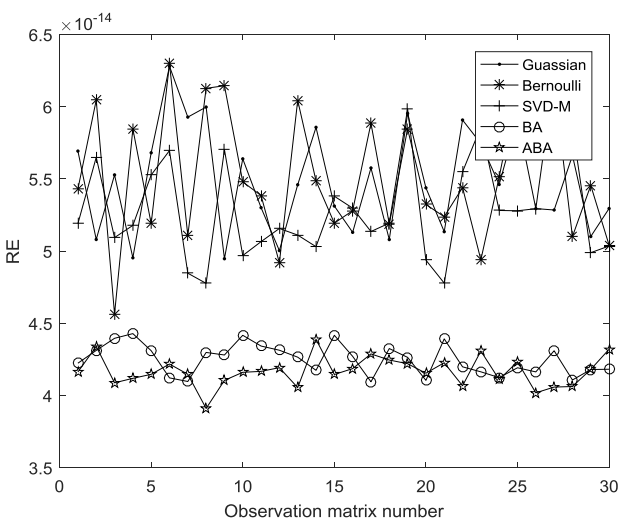

(e)

Figure 6. Reconstruction error of each algorithm to signal-3 with different compression ratio: (a) Rate $=0.3 ;(\mathbf{b})$ Rate $=0.35 ;(\mathbf{c})$ Rate $=0.4 ;(\mathbf{d})$ Rate $=0.45 ;(\mathbf{e})$ Rate $=0.5$.

Figure 6 shows the reconstructed error values obtained for the signals $S_{3}$ under each observation matrix and algorithm. It can be seen from Figure 6 the poor reconstruction error and the larger error value for Gaussian, Bernoulli, and SVD-M. For BA and ABA, the reconstruction effects are significantly better than the observation matrices.

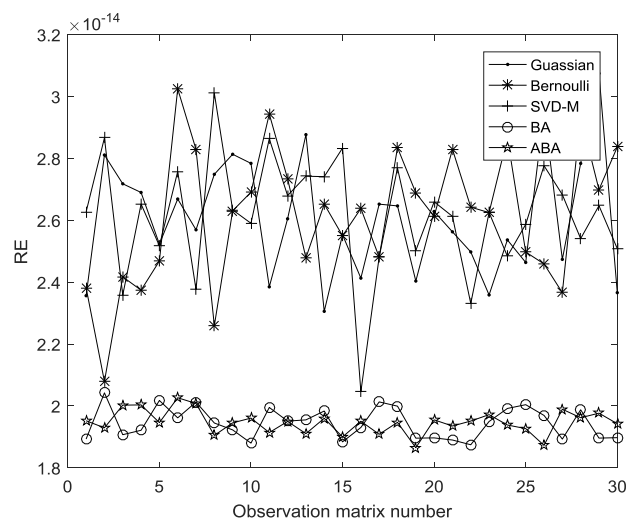

(a)

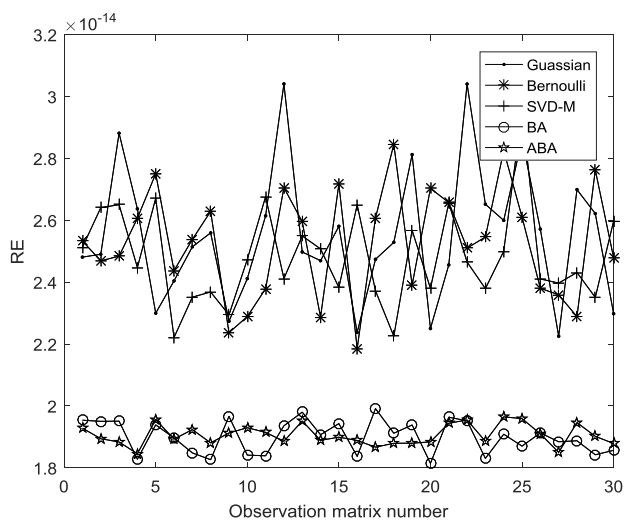

(b)

Figure 7. Cont. 


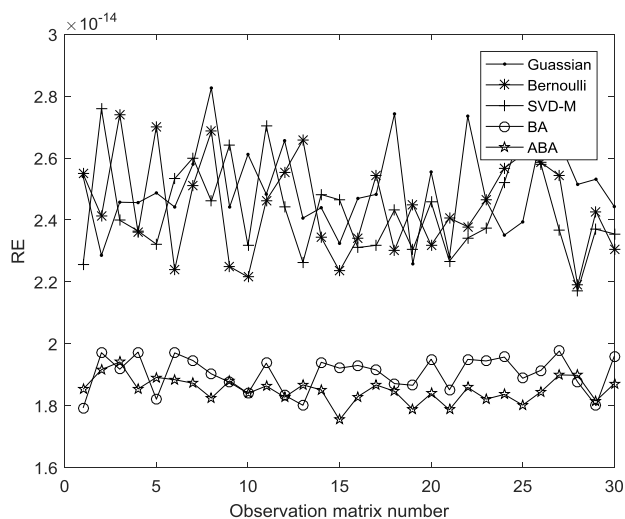

(c)

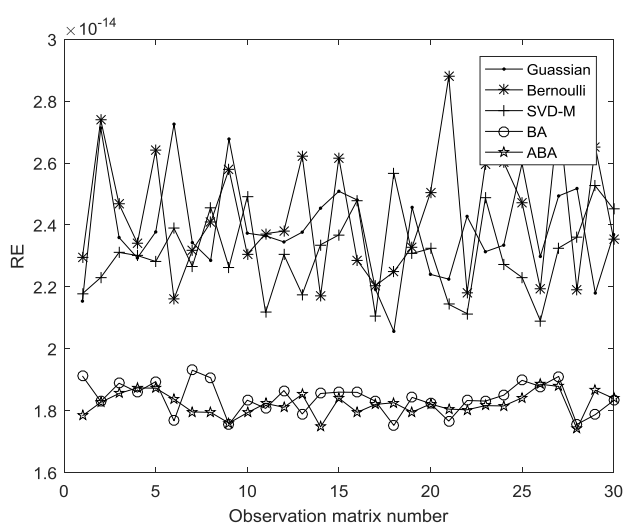

(d)

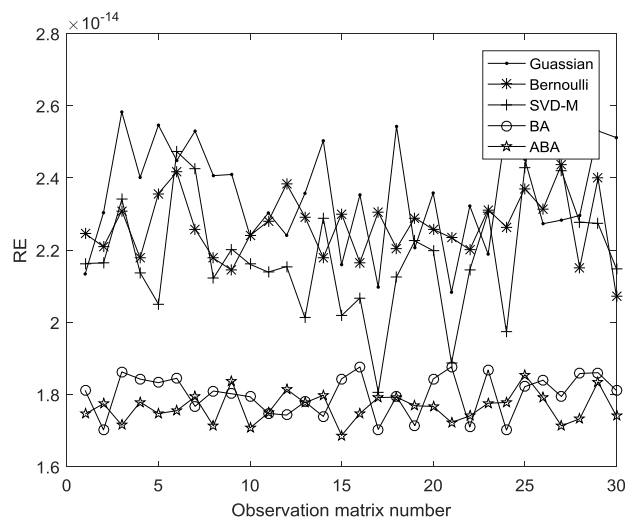

(e)

Figure 7. Reconstruction error of each algorithm to signal-4 with different compression ratio: (a) Rate $=0.3 ;(\mathbf{b})$ Rate $=0.35 ;(\mathbf{c})$ Rate $=0.4 ;(\mathbf{d})$ Rate $=0.45 ;(\mathbf{e})$ Rate $=0.5$.

Figure 7 illustrates the reconstruction error plots for several observation matrices and algorithms for the $S_{4}$ signals. Comparing the BA and ABA, the reconstruction error values and stability of Gaussian, Bernoulli, and SVD-M are poor, which also shows that the reconstruction effects of BA and ABA are obviously better from Figure 7. Moreover, it can be seen from the above Figures 4-7 that the ABA has better stability and error value than the BA algorithm.

\subsection{Analysis and Selection of Transformation Basis}

The selection of the transform base, as the preliminaries of the compressed sensing process, has a great influence on the signal reconstruction effect. A good transform base can well represent the original signal containing a large amount of information, and obtain a better reconstruction algorithm. Therefore, to get better transform bases, this section performs signal reconstruction experiments by comparing different transform bases. In addition, it proves the robustness of the ABA to optimize the observation matrix relative to different transformation basis.

\subsubsection{Transform Base Setting}

In this section, the transformation bases involved are as follows:

- Discrete Hartley transform matrix [68] (DHT),

- Discrete Fourier transform matrix [69] (FFT). 


\subsubsection{Evaluation Index Design}

The performance improvement rate PI is used to improve the reconstruction effect, the calculation Equation (13) is as follows.

$$
P I_{j}=\frac{\mid \text { Mean }_{0}-\text { Mean }_{j} \mid}{\operatorname{Mean}_{j}}
$$

where $\mathrm{Mean}_{0}$ represents the average fitness value of proposed algorithm, Mean ${ }_{j}$ represents the average fitness value of comparison algorithm $j$. In addition, the experimental parameters are set as in Section 4.1.3, another indicator (RE) is calculated as shown in Section 4.2.1 and the fitness function is shown in Equation (6).

\subsubsection{Selection of Transform Bases in Signal Reconstruction Experiments}

In signal reconstruction experiments, signal reconstruction experiments are performed on signals $S_{1}$ and $S_{3}$ with different transform bases. And the observation matrix used is the same as the ABA optimized observation matrix. The experimental results are shown in Table 3.

Table 3. Reconstruction effect of different transform based on signal.

\begin{tabular}{|c|c|c|c|c|}
\hline Signal & Rate & Data & DHT & FFT \\
\hline \multirow{5}{*}{$S_{1}$} & 0.30 & mean & $3.6916 \times 10^{-15}$ & $3.1462 \times 10^{-15}$ \\
\hline & 0.35 & mean & $3.4521 \times 10^{-15}$ & $3.1030 \times 10^{-15}$ \\
\hline & 0.40 & mean & $3.4700 \times 10^{-15}$ & $3.1139 \times 10^{-15}$ \\
\hline & 0.45 & mean & $3.3844 \times 10^{-15}$ & $3.0513 \times 10^{-15}$ \\
\hline & 0.50 & mean & $3.3120 \times 10^{-15}$ & $3.0155 \times 10^{-15}$ \\
\hline \multirow{5}{*}{$S_{3}$} & 0.30 & mean & $5.0519 \times 10^{-14}$ & $4.6387 \times 10^{-14}$ \\
\hline & 0.35 & mean & $4.9979 \times 10^{-14}$ & $4.4886 \times 10^{-14}$ \\
\hline & 0.40 & mean & $4.8152 \times 10^{-14}$ & $4.3008 \times 10^{-14}$ \\
\hline & 0.45 & mean & $4.7886 \times 10^{-14}$ & $4.2642 \times 10^{-14}$ \\
\hline & 0.50 & mean & $4.7102 \times 10^{-14}$ & $4.1672 \times 10^{-14}$ \\
\hline
\end{tabular}

The bold indicates that signals can be reconstructed with better results under different observation matrices and transform bases.

It can be seen from Table 3 that DHT and FFT reconstruction are successful. Furthermore, both signal reconstruction errors are superior to DHT transform bases when using FFT transform bases. Therefore, the FFT transform base is more suitable for signal reconstruction. In this paper, we select the FFT transform basis as the signal sparse tool.

\subsubsection{Analysis of the Influence of Transformation Basis on ABA}

The correlation between the transformation base and the observation matrix directly affects the reconstruction effect. Gaussian matrix is not related to most transform bases [70]. Therefore, our experiments employ Gaussian matrix as the observation matrix to prove the robustness of the ABA algorithm to optimize the observation matrix with different transform bases.

In the signal reconstruction experiment, to test the effectiveness of different observation matrices with different transform bases, we take the $S_{1}$ and $S_{3}$ signals and tests the effects of different observation matrices under transform bases DHT and FFT. According to the proposed evaluation index reconstruction error (RE) and performance improvement rate (PI) values, the optimization effect of the observation matrix is reflected, and the smaller mean and reconstruction error are selected as the optimal values, and then the conversion comparison is performed. The selection and reconstruction 
effects of different transform bases based on adaptive bat algorithm are compared, and the robustness of the algorithm is fully proved. The results of the experiment are shown in Table 4.

Table 4. Reconstruction effect of different observation matrices and transform bases.

\begin{tabular}{|c|c|c|c|c|c|c|}
\hline \multirow{2}{*}{ Signal } & \multirow{2}{*}{ Rate } & \multirow{2}{*}{ Data } & \multicolumn{2}{|c|}{ DHT } & \multicolumn{2}{|c|}{ FFT } \\
\hline & & & Gaussian & ABA & Gaussian & ABA \\
\hline \multirow{10}{*}{$S_{1}$} & \multirow{2}{*}{0.30} & $R E$ & $4.5714 \times 10^{-14}$ & $3.6916 \times 10^{-14}$ & $4.0586 \times 10^{-15}$ & $3.1462 \times 10^{-15}$ \\
\hline & & $P I$ & $19.25 \%$ & - & $22.48 \%$ & - \\
\hline & \multirow{2}{*}{0.35} & $R E$ & $4.3217 \times 10^{-14}$ & $3.4521 \times 10^{-14}$ & $3.8985 \times 10^{-15}$ & $3.1030 \times 10^{-15}$ \\
\hline & & $P I$ & $20.12 \%$ & - & $20.41 \%$ & - \\
\hline & \multirow{2}{*}{0.40} & $R E$ & $4.2001 \times 10^{-14}$ & $3.4700 \times 10^{-14}$ & $3.8786 \times 10^{-15}$ & $3.1139 \times 10^{-15}$ \\
\hline & & PI & $17.38 \%$ & - & $19.72 \%$ & - \\
\hline & \multirow{2}{*}{0.45} & $R E$ & $4.1711 \times 10^{-14}$ & $3.3844 \times 10^{-14}$ & $3.8565 \times 10^{-15}$ & $3.0513 \times 10^{-15}$ \\
\hline & & PI & $18.86 \%$ & - & $20.88 \%$ & - \\
\hline & \multirow{2}{*}{0.50} & $R E$ & $4.1014 \times 10^{-14}$ & $3.3120 \times 10^{-14}$ & $3.8174 \times 10^{-15}$ & $3.0155 \times 10^{-15}$ \\
\hline & & PI & $19.25 \%$ & - & $21.01 \%$ & - \\
\hline \multirow{10}{*}{$S_{3}$} & \multirow{2}{*}{0.30} & $R E$ & $6.5552 \times 10^{-14}$ & $5.0519 \times 10^{-14}$ & $6.1340 \times 10^{-15}$ & $4.6387 \times 10^{-15}$ \\
\hline & & PI & $22.93 \%$ & - & $24.38 \%$ & - \\
\hline & \multirow{2}{*}{0.35} & $R E$ & $6.5499 \times 10^{-14}$ & $4.9979 \times 10^{-14}$ & $5.9468 \times 10^{-15}$ & $4.4886 \times 10^{-15}$ \\
\hline & & $P I$ & $23.70 \%$ & - & $24.50 \%$ & - \\
\hline & \multirow{2}{*}{0.40} & $R E$ & $6.4900 \times 10^{-14}$ & $4.8152 \times 10^{-14}$ & $5.7716 \times 10^{-15}$ & $4.3008 \times 10^{-15}$ \\
\hline & & PI & $25.81 \%$ & - & $25.48 \%$ & - \\
\hline & \multirow{2}{*}{0.45} & $R E$ & $6.4718 \times 10^{-14}$ & $4.7886 \times 10^{-14}$ & $5.6797 \times 10^{-15}$ & $4.2642 \times 10^{-15}$ \\
\hline & & PI & $26.01 \%$ & - & $24.92 \%$ & - \\
\hline & \multirow{2}{*}{0.50} & $R E$ & $6.4152 \times 10^{-14}$ & $4.7102 \times 10^{-14}$ & $5.4878 \times 10^{-15}$ & $4.1672 \times 10^{-15}$ \\
\hline & & PI & $26.58 \%$ & - & $24.06 \%$ & - \\
\hline
\end{tabular}

The bold indicates that signals can be reconstructed with better results under different observation matrices and transform bases.

It can be seen from Table 4 that the reconstruction effect of the ABA optimization observation matrix is optimal for different transform bases. Moreover, the reconstructed error values are equivalent by comparing the two transform bases, which proves the feasibility of the ABA on different transform bases in the signal reconstruction experiment.

\section{Conclusions}

As we know, it is critical to select an appropriate observation matrix to ensure high signal reconstruction accuracy. In this paper, an adaptive bat algorithm is proposed to optimize the observation matrix. To improve the global search ability, the position of the optimal solution is updated continuously by designing the adaptive bat algorithm search method. Further, the adaptive bat algorithm is used to optimize the observation matrix. Finally, we verify our model on four different signals, the optimized observation matrix and the smaller reconstruction error value are obtained. The simulation results fully show that the adaptive bat algorithm optimization observation matrix can obtain higher signal reconstruction accuracy and better robustness, compared with other algorithms.

Author Contributions: Formal analysis, Z.C. and Y.Z.; Funding acquisition, Z.C.; Investigation, Y.Z. and C.Z.; Methodology, Z.C. and C.Z.; Project administration, Z.C.; Supervision, Z.C. and Z.S.; Visualization, Z.C. and C.Z.; Writing—original draft, Y.Z.; Writing—review \& editing, Y.Z. 
Funding: This work is supported by the National Natural Science Foundation of China under Grant No.61806138, No. U1636220 and No.61663028, Natural Science Foundation of Shanxi Province under Grant No.201801D121127, PhD Research Startup Foundation of Taiyuan University of Science and Technology under Grant No.20182002.

Conflicts of Interest: The authors declare no conflict of interest.

\section{References}

1. Gilbert, A.C.; Guha, S.; Indyk, P.; Muthukrishnan, S.; Strauss, M. Near-optimal sparse Fourier representations via sampling. In Proceedings of the 34th ACM Symposium on Theory of Computing, Montreal, QC, Canada, 19-21 May 2002; pp. 152-161.

2. Candes, E.; Romberg, J.; Tao, T. Robust Uncertainty Principles: Exact Signal Reconstruction from Highly Incomplete Frequency Information. arXiv 2004, arXiv:0409186. [CrossRef]

3. Donoho, D.L. Compressed sensing. IEEE Trans. Inf. Theory 2006, 52, 1289-1306. [CrossRef]

4. Bloom, W.R. Estimates for the Fourier transform. Math. Sci. 1985, 10, 65-81.

5. Wang, Q.; Zhang, P.; Wang, H.; Yang, W.; Chen, Y. Survey of Measurement Matrix Construction in Compressed Sensing. Comput. Appl. 2017, 37, 188-196. [CrossRef]

6. Candes, E.J.; Tao, T. Near-Optimal Signal Recovery from Random Projections: Universal Encoding Strategies. IEEE Trans. Inf. Theory 2004, 52, 5406-5425. [CrossRef]

7. Donoho, D.L.; Tsaig, Y.; Drori, I.; Starck, J.L. Sparse Solution of Underdetermined Systems of Linear Equations by Stagewise Orthogonal Matching Pursuit. IEEE Trans. Inf. Theory 2012, 58, 1094-1121. [CrossRef]

8. Fong, H.; Zhang, Q.; Wei, S. Image Reconstruction Method Based on Sub-gaussian Random Projection. Comput. Res. Dev. 2008, 45, 1402-1407.

9. Candes, E.J. The Restricted Isometry Property and its Implications for Compressed Sensing. Comptes Rendus-Mathématique 2008, 346, 589-592. [CrossRef]

10. Yin, W. Practical Compressive Sensing with Toeplitz and Circulant Matrices; SPIE-The International Society for Optical Engineering: Bellingham, WA, USA, 2010; p. 7744.

11. Peng, Y.; He, B.; Lin, Y. Compressed Sensing Noise Signal Reconstruction Algorithm Based on Singular Value Decomposition. J. Instrum. 2012, 33, 2655-2660.

12. Duarte-Carvajalino, J.M.; Sapiro, G. Learning to Sense Sparse Signals: Simultaneous Sensing Matrix and Sparsifying Dictionary Optimization. IEEE Trans. Image Process. 2009, 18, 1395-1408. [CrossRef]

13. Abolghasemi, V.; Jarchi, D.; Sanei, S. A robust approach for optimization of the measurement matrix in Compressed Sensing. In International Workshop on Cognitive Information Processing; IEEE: Piscataway, NJ, USA, 2010; pp. 388-392.

14. Lan, Y.; Wang, D.; Zheng, Q.; Zhai, M. Observation Matrix Optimization Based on Gradient Descent Method and QR Decomposition. Comput. Technol. Dev. 2017, 27, 190-194.

15. Yu, Y.; Petropulu, A.P.; Poor, H.V. Measurement Matrix Design for Compressive Sensing-Based MIMO Radar. IEEE Trans. Signal Process. 2011, 59, 5338-5352. [CrossRef]

16. Hoque, M.T.; Iqbal, S. Genetic algorithm-based improved sampling for protein structure prediction. Int. J. Bio-Inspired Comput. 2017, 9, 129-141. [CrossRef]

17. Jie, W.; Jiangjun, Y. A high-efficient multi-deme genetic algorithm with better load-balance. Int. J. Comput. Sci. Math. 2018, 9, 240-246. [CrossRef]

18. Cai, X.; Gao, X.Z.; Xue, Y. Improved bat algorithm with optimal forage strategy and random disturbance strategy. Int. J. Bio-Inspired Comput. 2016, 8, 205-214. [CrossRef]

19. Cui, Z.; Li, F.; Zhang, W. Bat algorithm with principal component analysis. Int. J. Mach. Learn. Cybern. 2019, 10, 603-622. [CrossRef]

20. Pooja, P.; Chaturvedi, P.; Kumar, A.; Tomar, A. A novel differential evolution approach for constraint optimization. Int. J.Bio-Inspired Comput. 2018, 12, 254-265. [CrossRef]

21. Chen, L.; Zhou, C.; Li, X.; Dai, G. An improved differential evolution algorithm based on suboptimal solution mutation. Int. J. Comput. Sci. Math. 2017, 8, 28-34. [CrossRef]

22. Lv, L.; Fan, T.; Li, Q.; Sun, Z.; Xu, L. Object tracking with improved firefly algorithm. Int. J. Comput. Sci. Math. 2018, 9, 219-231. [CrossRef]

23. Yaghoobi, T.; Esmaeili, E. An improved artificial bee colony algorithm for global numerical optimization. Int. J. Bio-Inspired Comput. 2017, 9, 251-258. [CrossRef] 
24. You, X.; Ma, Y.; Liu, Z. An improved artificial bee colony algorithm for solving parameter identification problems. Int. J. Comput. Sci. Math. 2017, 8, 570-579. [CrossRef]

25. Zhang, M.; Wang, H.; Cui, Z.; Chen, J. Hybrid multi-objective cuckoo search with dynamical local search. Memetic Comput. 2018, 10, 199-208. [CrossRef]

26. Cui, Z.; Sun, B.; Wang, G.; Xue, Y.; Chen, J. A novel oriented cuckoo search algorithm to improve DV-Hop performance for cyber-physical systems. J. Parallel Distrib. Comput. 2017, 10, 42-52. [CrossRef]

27. Abdel-Baset, M.; Zhou, Y.; Ismail, M. An improved cuckoo search algorithm for integer programming problems. Int. J. Comput. Sci. Math. 2018, 9, 66-81. [CrossRef]

28. Cui, Z.; Zhang, J.; Wang, Y.; Cao, Y.; Cai, X.; Zhang, W.; Chen, J. A pigeon-inspired optimization algorithm for many-objective optimization problems. Sci. China Inf. Sci. 2019, 62, 070212. [CrossRef]

29. Li, J.; Ke, L.; Ye, G.; Zhang, T. Ant colony optimization for the routing problem in the constellation network with node satellite constraint. Int. J. Bio-Inspired Comput. 2017, 10, 267-274. [CrossRef]

30. Pérez-Delgado, M.L. An iterative method to improve the results of ant-tree algorithm applied to colour quantisation. Int. J. Bio-Inspired Comput. 2018, 12, 87-114. [CrossRef]

31. Cai, X.; Wang, P.; Du, L.; Cui, Z.; Zhang, W.; Chen, J. Multi-objective 3-Dimensional DV-Hop Localization Algorithm with NSGA-II. IEEE Sens. J. 2019. [CrossRef]

32. Wang, P.; Huang, J.; Cui, Z.; Xie, L.; Chen, J. A Gaussian Error Correction Multi-Objective Positioning Model with NSGA-II. Concurr. Comput. Pract. Exp. 2019. [CrossRef]

33. Cui, Z.; Du, L.; Wang, P.; Cai, X.; Zhang, W. Malicious code detection based on CNNs and multi-objective algorithm. J. Parallel Distrib. Comput. 2019, 129, 50-58. [CrossRef]

34. Cui, Z.; Chang, Y.; Zhang, J.; Cai, X.; Zhang, W. Improved NSGA-III with selection-and-elimination operator. Swarm Evol. Comput. 2019, 49, 23-33. [CrossRef]

35. Wang, G.G.; Cai, X.; Cui, Z.; Min, G.; Chen, J. High Performance Computing for Cyber Physical Social Systems by Using Evolutionary Multi-Objective Optimization Algorithm; IEEE Transactions on Emerging Topics in Computing: Piscataway, NJ, USA, 2017. [CrossRef]

36. Zhang, J.; Xue, F.; Cai, X.; Cui, Z.; Chang, Y.; Zhang, W.; Li, W. Privacy protection based on many-objective optimization algorithm. In Concurrency and Computation Practice and Experience; John Wiley \& Sons: Hoboken, NJ, USA, 2019. [CrossRef]

37. Wang, P.; Xue, F.; Li, H.; Cui, Z.; Chen, J. A multi-objective DV-Hop localization algorithm based on NSGA-II in internet of things. Mathematics 2019, 7, 184. [CrossRef]

38. Sadeghiram, S. Bacterial foraging optimisation algorithm, particle swarm optimisation and genetic algorithm: A comparative study. Int. J. Bio-Inspired Comput. 2017, 10, 275-282. [CrossRef]

39. Cortés, P.; Muñuzuri, J.; Onieva, L.; Guadix, J. A discrete particle swarm optimisation algorithm to operate distributed energy generation networks efficiently. Int. J. Bio-Inspired Comput. 2018, 12, 226-235. [CrossRef]

40. Arloff, W.; Schmitt, K.R.; Venstrom, L.J. A parameter estimation method for stiff ordinary differential equations using particle swarm optimization. Int. J. Comput. Sci. Math. 2018, 9, 419-432. [CrossRef]

41. Zhang, J.; Jie, J.; Wang, W.; Xu, X. A hybrid particle swarm optimisation for multi-objective flexible job-shop scheduling problem with dual-resources constrained. Int. J. Comput. Sci. Math. 2017, 8, 526-532. [CrossRef]

42. Cui, Z.; Cao, Y.; Cai, X.; Cai, J.; Chen, J. Optimal LEACH protocol with modified bat algorithm for big data sensing systems in Internet of Things. J. Parallel Distrib. Comput. 2019, 132, 217-229. [CrossRef]

43. Wang, H.Q.; Yang, B.L.; Qin, A.H. An encoding and reconstructing method with robust transmission for 3D model topological data over wireless network. Int. J. Comput. Sci. Math. 2017, 8, 542-551. [CrossRef]

44. Tripathi, A.; Saxena, N.; Mishra, K.K.; Misra, A.K. A nature inspired hybrid optimisation algorithm for dynamic environment with real parameter encoding. Int. J. Bio-Inspired Comput. 2017, 10, 24-32. [CrossRef]

45. Cai, X.; Wang, H.; Cui, Z.; Cai, J.; Xue, Y.; Wang, L. Bat algorithm with triangle-flipping strategy for numerical optimization. Int. J. Mach. Learn. Cybern. 2018, 9, 199-215. [CrossRef]

46. Ren, Y.; Sun, Y.; Jing, X.; Cui, Z.; Shi, Z. Adaptive Makeup Transfer via Bat Algorithm. Mathematics 2019, 7, 273. [CrossRef]

47. Cui, Z.; Zhang, C.; Shi, Z. Observation Matrix Optimization Algorithm Based on Bat Algorithm. Control Decis. Mak. 2018, 33, 192-195.

48. Fang, H.; Yang, H. Greedy algorithm and compressed sensing theory. J. Autom. 2011, 37, 1413-1421. 
49. Bi, H.; Zhao, C.; Liu, Y.; Li, N. Performance evaluation of greedy reconstruction algorithms in compressed sensing. In Proceedings of the 9th International Congress on Image and Signal Processing, BioMedical Engineering and Informatics (CISP-BMEI); IEEE: Piscataway, NJ, USA, 2017; pp. 1322-1327.

50. Yang, A.Y.; Sastry, S.S.; Ganesh, A.; Ma, Y. Fast 11-minimization algorithms and an application in robust face recognition: A review. In IEEE International Conference on Image Processing; IEEE: Piscataway, NJ, USA, 2010; pp. 1849-1852.

51. Zhao, H.; Chen, J.; Xu, S.; Wang, Y.; Qiao, Z. Compressive sensing for noisy solder joint imagery based on convex optimization. Solder. Surf. Mt. Technol. 2016, 28, 114-122. [CrossRef]

52. Chartrand, R.; Yin, W. Iteratively reweighted algorithms for compressive sensing. In IEEE International Conference on Acoustics, Speech and Signal Processing; IEEE: Piscataway, NJ, USA, 2008; pp. 3869-3872.

53. You, G.; Huang, Z.H.; Wang, Y. A Theoretical Perspective of Solving Phaseless Compressive Sensing via Its Nonconvex Relaxation. Inf. Sci. 2017, 11, 254-268. [CrossRef]

54. Liu, F.; Lin, L.; Jiao, L.; Li, L.; Yang, S.; Hou, B.; Xu, J. Nonconvex compressed sensing by nature-inspired optimization algorithms. IEEE Trans. Cybern. 2015, 45, 1042-1053.

55. Li, H.; Su, X.; Xu, Z.; Zhang, Q. MOEA/D with Iterative Thresholding Algorithm for Sparse Optimization Problems. In Parallel Problem Solving from Nature_PPSN XII; Springer: Berlin/Heidelberg, Germany, 2012; pp. 93-101.

56. Hou, K. Research on Reconstruction Algorithm Based on Compressed Sensing; Chongqing University: Chongqing, China, 2013.

57. Cai, T.T.; Wang, L. Orthogonal Matching Pursuit for Sparse Signal Recovery with Noise. IEEE Trans. Inf. Theory 2011, 57, 4680-4688. [CrossRef]

58. Mirjalili, S.; Mirjalili, S.M.; Yang, X.S. Binary bat algorithm. Neural Comput. Appl. 2014, 25, 663-681. [CrossRef]

59. Osaba, E.; Yang, X.S.; Diaz, F.; Lopez-Garcia, P.; Carballedo, R. An improved discrete bat algorithm for symmetric and asymmetric Traveling Salesman Problems. Eng. Appl. Artif. Intell. 2016, 48, 59-71. [CrossRef]

60. Hua, X.; Ting, Z. Hybrid discrete bat algorithm for multi-objective flexible job shop scheduling. J. Mech. Eng. 2016, 52, 201-212.

61. Liu, C.; Ye, C. Bat algorithm with Levy flight characteristics. J. Intell. Syst. 2013, 3, 240-246.

62. Xie, J.; Zhou, Y.; Chen, H. A bat algorithm based on levy flight trajectory. Intell. Mode Artif. Intell. 2013, 26, 829-837.

63. Wang, C.; Ma, M.; Shen, P. A New Improved Bat Algorithm for Global Optimization. Math. Appl. 2016, 29, 632-642. [CrossRef]

64. Tsai, P.W.; Pan, J.S.; Liao, B.Y.; Tsai, M.J.; Istanda, V. Bat Algorithm Inspired Algorithm for Solving Numerical Optimization Problems. Appl. Mech. Mater. 2012, 148, 134-137. [CrossRef]

65. Yang, X. Bat algorithm for multi-objective optimization. Int. J. Bio-Inspired Comput. 2012, 3, $267-274$. [CrossRef]

66. Wang, Y.; Jia, C.; Zhao, R. Multi-objective bat algorithm based on decomposition. J. Agric. Mach. /Trans. Chin. Soc. Agric. Mach. 2015, 46, 316-324.

67. Heraguemi, K.E.; Kamel, N.; Drias, H. Multi-objective Bat Algorithm for Mining Interesting Association Rules. Int. J. Bio-Inspired Comput. 2016, 11, 239. [CrossRef]

68. He, D.J.; Jiang, P. Research on Fast Image Matching Method Based on Discrete Hartley Transform. Mod. Def. Technol. 2016, 44, 61-65.

69. Wang, Q.; Li, J.; Shen, Y. A review of algorithms for constructing deterministic measurement matrix in compressed sensing. Electron. J. 2013, 41, 2041-2050.

70. Yang, Z. Compressed Sensing Reconstruction Technology and Its Application in Image Fusion; Nanjing University of Posts and Telecommunications: Nanjing, China, 2014.

(C) 2019 by the authors. Licensee MDPI, Basel, Switzerland. This article is an open access article distributed under the terms and conditions of the Creative Commons Attribution (CC BY) license (http://creativecommons.org/licenses/by/4.0/). 